\title{
COMPARAÇÃO DA CAPACIDADE FUNCIONAL ENTRE OBESOS, PÓS TARDIO DE CIRURGIA BARIÁTRICA
}

\author{
Amanda F. da Silva', Arthur F. Gimenes,2, Narana M. de Souza², Débora Benfeita Amaral', \\ Millena A. O. da Silva², Dayana B. Araújo', Gabrielly De A. Pinto ${ }^{2} \&$ Luciano M. Chicayban $^{1}$
}

(1) Pesquisador do Laboratório de Análises de Disfunções Pneumofuncionais (LADPF/ISECENSA) - Institutos Superiores de Ensino do CENSA ISECENSA, Rua Salvador Correa, 139, Centro, Campos dos Goytacazes, RJ, Brasil; (2) Aluno (a) voluntário (a) de Iniciação Científica do PROVIC/ISECENSA.

A obesidade é um distúrbio metabólico, considerado uma epidemia mundial e um grande problema da saúde pública. A obesidade relaciona-se com comorbidades como hipertensão, diabetes mellitus tipo II, doenças cardiovasculares, restrição ventilatória, contribuindo para a redução da capacidade funcional e aumento da mortalidade. O objetivo deste estudo foi comparar a capacidade funcional entre pacientes obesos, pós cirurgia bariátrica e eutróficos. Foi realizado um estudo observacional transversal com 27 voluntários de ambos os sexos, em 3 grupos. Obesos grau II e III $(\mathrm{N}=7)$; pós tardio de cirurgia bariátrica $(\mathrm{N}=10)$ e eutróficos $(\mathrm{N}=10)$. Os indivíduos com doenças respiratórias prévias, doenças osteomioarticulares ou com contra-indicação a prática de exercícios foram excluídos. A capacidade funcional foi avaliada através do teste de caminhada de 6 minutos TC6, com a monitorização da distância percorrida (DTC6), frequência cardíaca (FC), pressão arterial (PA), escala de Borg e saturação periférica de oxigênio (SpO2) ao longo do teste. Foi considerado significativo $\mathrm{p}<0,05$. No repouso e sob mesmo nível de esforço submáximo (no sexto minuto do teste), o grupo de obesos apresentou maior frequência cardíaca (FC) e pressão arterial sistólica (PAS), na comparação com o grupo pós bariátrica $(\mathrm{p}=0,002)$ e eutróficos $(\mathrm{p}<0,001)$. Além disso, os obesos apresentaram maior frequência respiratória $(\mathrm{FR})$ ao repouso $(\mathrm{p}<0,05)$. $\mathrm{O}$ grupo de obesos percorreu menor distância no TC6 em comparação com os pós-bariátrica, e estes menos em comparação com os eutróficos $(\mathrm{p}<0,05)$. Conclui-se que os indivíduos obesos apresentaram menor capacidade funcional, comparado com os eutróficos e pós bariátrica, além de maior FC e PAS ao repouso e para o mesmo nível de esforço submáximo.

Palavras-chave: Fisioterapia, Cirurgia Bariátrica, Obesidade. 